\title{
Adenoid cystic carcinoma of the maxilla
}

\author{
Arghya Basu', Jagjit Kumar Pandey ${ }^{2}$, Arnab Mondal ${ }^{2}$ \\ ${ }^{1}$ Department of Oncology, B. R. Singh Hospital \& Research Centre, Kolkata, West Bengal, India \\ ${ }^{2}$ Department of surgical oncology, Chittaranjan National Cancer Institute, Kolkata, India
}

Key words: Adenoid cystic carcinoma; carcinoma of maxilla; maxillectomy; perineural spread

\section{Introduction}

Adenoid cystic carcinomas (ACC) are malignant tumours of secretory glands mainly affecting major and minor salivary glands of the head and neck. It also involves lacrimal glands, ceruminous glands and occasionally female genital excretory glands. Although palate, the floor of the mouth and buccal mucosa are commonly affected, uncommon sites like lungs (tracheobronchial tree), oesophagus, also been reported [1].

ACCs are the malignancy in lacrimal glands and 2nd most common malignancy in salivary glands. They are well known for the prolonged unpredictable clinical course and the tendency of delayed metastasis. Lungs are the most commonly involved metastatic sites followed by bone, brain, kidney and liver. Tumour size, grade, stage, lymph node involvement, neural invasion and margin status are the most important prognostic factors [2]. Long-term survival can be achieved by a combination of surgery and radiotherapy. Immunohistochemistry prevents misdiagnosis and helps to determine appropriate multimodality management protocol. Here we present a rare case of adenoid cystic carcinoma affecting the palate and maxillary sinus which was managed with surgery and radiotherapy at Chittaranjan National Cancer Institute, Kolkata, and the centre of Eastern India.

\section{Case report}

A 77 years old male presented with slowly progressing hard bony swelling in right half of roof of the mouth for several years but recently increasing in size during the last 3 months. He was a regular smoker for last 55 years. but not alcoholic. He gave the history of nose blockade for the last 3 months with numbness over the right side of upper lip. Extra oral examination showed the fullness of the right maxillary region without any cervical lymphadenopathy. Intraoral examination showed an oval-shaped solitary, non-tender, firm, fixed, erythematous mass with diffuse borders in right

Correspondence: Arghya Basu

E-mail: dr.arghyarox@gmail.com

Received: 25-03-2018 Accepted: 29-09-2018

(iD) http://orcid.org/0000-0003-4074-6547

DOI: http://doi.org/10.4038/sljs.v36i3.8532

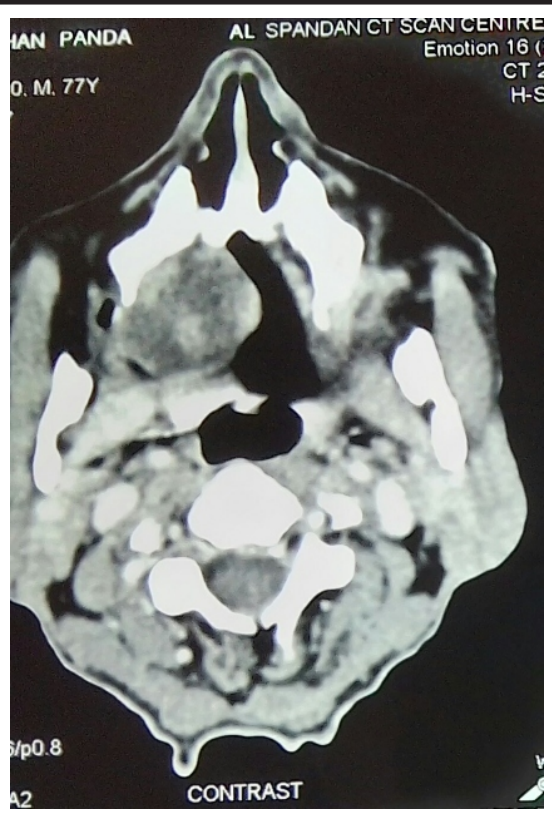

Figure 1.CT Scan showing the tumour

maxilla involving hard palate and extending anteriorly from palatal rughae to posteriorly up to soft palate, medially $1 \mathrm{~cm}$ away from midline to laterally from lateral incisor to 1 st molar tooth without any mucosal ulceration. Teeth in relation to the swelling were non-mobile / non-carious / non-tender.

Panoramic radiograph showed a unilocular radiolucency obliterating the right maxillary sinus and involving the cortical plate above the lesion. Contrast-enhanced CT scan showed an enhanced $5 \times 4.5 \times 3 \mathrm{~cm}$ mass in right maxilla with the destruction of maxillary sinus and involvement of hard palate. Fat-suppressed T1 weighted MRI with gadolinium enhancement showed a high-density tumour in right palatal region extending to the buccal region of the alveolar bone in coronal section and a high-intensity area on right half of hard palate with the involvement of the major palatine nerve in axial section. No cervical lymphadenopathy, metastatic site or any other second primary was seen radiologically.

Incisional biopsy showed monomorphic cells arranged in the glandular and canalicular pattern with focal areas of cribriforming with mucin-like material inside. Fibrous stroma was seen with areas of hyalinization and islands of uniform cells arranged in the cord-like pattern with hyperchromatic 


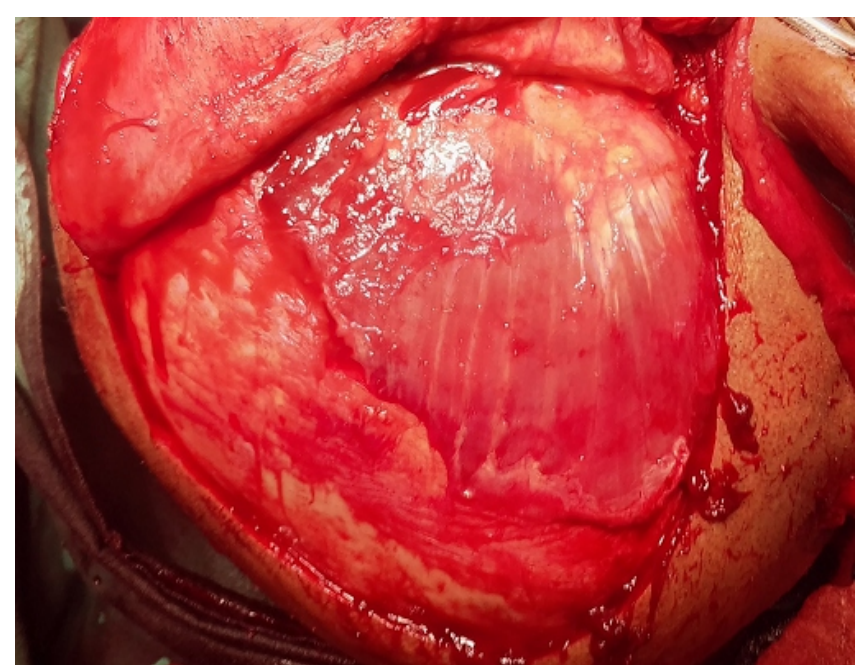

Figure 2. Intraoperative photo

nuclei enclosing round to oval pseudocystic spaces containing eosinophilic coagulum. Immunohistochemistry showed positive reaction pattern to S-110 (diffusely cytoplasmic and nuclear staining), pan-cytokeratin (AE1/AE3) (cell membrane staining), Vimentin, p53, C-Kit, Alpha Smooth Muscle Actin , p63 and a ki 67 labelling index of $60 \%$ (nuclear staining). CD 117 was strongly positive confirming the cribriform type of ACC. The patient was treated with right-sided subtotal maxillectomy followed by reconstruction with temporalis muscle flap. Histopathology showed adenoid cystic carcinoma with a clear margin, no lymphovascular invasion was identified but the presence of perineural invasion was noticed. The maxillary cavity was treated with adjuvant radiotherapy of 60 Gy delivered in 30 fractions. The patient is surviving for 2 years now without any locoregional or distant recurrence.

\section{Discussion}

ACCs were first described by Bilroth in 1856 as 'Cylindroma' due to its characteristic histological appearance. It is a very rare malignancy accounting for $<1 \%$ of all head and neck malignancies and around $10 \%$ of all salivary gland neoplasms. ACCs show female predilection with a female: male ratio of 1.2: 1 . These are aggressive, relentless, slowly growing tumours with the insidious destruction of surrounding tissues and perineural invasion. Pain is often an early sign (even before the appearance of swelling) because of neurotropism. Perineural invasion is the pathognomonic factor responsible for high rate of local recurrence. Lymph node involvement is rare and is usually caused by contiguous spread rather than lymphatic permeation or embolization [3].

Three distinct patterns of ACCs have been described: solid, cribriform and tubuloductal, cribriform being the most common, solid having worst prognosis and highest recurrence rates. 5 years recurrence rates of $100 \%, 89 \%$ and $59 \%$ have been reported for these three patterns respectively [4]. Differential diagnoses are polymorphous low-grade

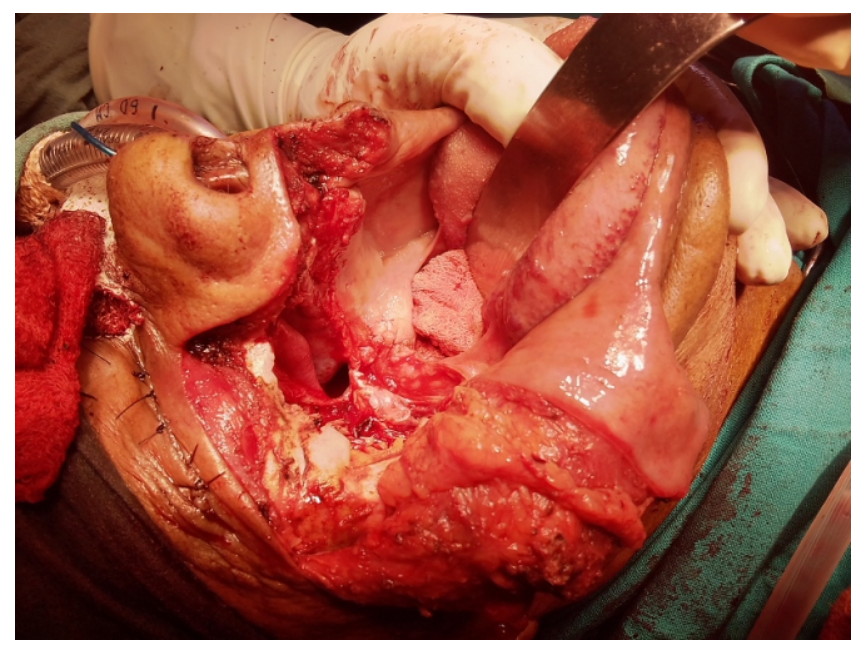

Figure 3. Harvesting of temporalis flap

adenocarcinoma (PLGA), basal cell adenoma (BCA), mixed tumour and basaloid squamous cell carcinoma (BSCC). Immunohistochemistry demonstrates pseudocysts positive for periodic acid Schiff (PAS) and alcian blue and contain basement membrane components like type IV collagen, heparin sulfate and laminin isoforms. Epithelial cells are positive for carcinoembryonic antigen (CEA) and epithelial membrane antigen (EMA), duct lining cells are positive for CD-117 and myoepithelial cells are positive for S-100 protein, calponin, p63, smooth muscle actin and myosin. Perineural invasion is often indicated by the presence of S100, glial fibrillary acidic protein (GFAP) and neural cell adhesion molecule whereas p53 is often involved with tumour progression and recurrence. High Ki-67 index and presence of more than $50 \%$ solid areas also indicate an aggressive clinical course. Alterations in chromosomes $6 \mathrm{q}, 9 \mathrm{p}$ and $17 \mathrm{p}$ 12-13 are most frequently observed [4].

CT and MRI are very useful to delineate a tumour, to plan extent of surgery and to look out for recurrences on postoperative follow-up, especially to determine the submucosal extent and perineural spread which can be difficult to assess clinically. The centre of the lesion usually presents with low density on $\mathrm{CT}$, a characteristic feature that can help to differentiate it from squamous cell carcinomas. The changes on MRI are characterized solely by an increase in intensity with the preservation of the shape.

Surgical excision with oncologically acceptable margins and adjuvant radiation remains the mainstay of treatment with a 5 and 10 years survival rate of $77 \%$ and $57 \%$ respectively. Chemotherapy is yet to find a role in ACCs. Neck dissection should be performed in presence of clinically and/or radiologically evident cervical lymphatic metastasis. Prognosis is highly dependent on a solid histological type, perineural spread, and distant metastasis and locoregional recurrences. Clinically and radiologically undetectable micro invasion of bones often leads to locoregional recurrence even 
after successful treatment completion. Bone metastases usually correspond to rapid tumour dissemination and death, whereas lung metastasis leads to a less aggressive clinical course. Hence, a close surveillance and long-term follow up is recommended to prevent local recurrence and distant metastasis [5].

\section{Conclusion}

ACCs are one of the most destructive and unpredictable malignancies with an indolent growth pattern. They often show aggressive behaviour, infiltrating into surrounding tissues and may present with regional lymphadenopathy and/or distant metastasis at the time of discovery. Early diagnosis and adequate surgical resection with adjuvant radiotherapy are the mainstays of treatment. Although our case did not have any recurrence disease for 2 years, a dose long-term follow up is needed, as $100 \%$ solid form of ACC could recur.

All authors disclose no conflict of interest. The study was conducted in accordance with the ethical standards of the relevant institutional or national ethics committee and the Helsinki Declaration of 1975, as revised in 2000 .

\section{References}

1. Chundru NSV, Amudala R, Thankappan P, Nagaraju CD . Adenoid cystic carcinoma of palate: A case report and review of literature . Dental Research Journal . doi: 10.4103/1735-3327.113372 .2013;10(2):274-278.

2. Rahmani K, Taghipour Zahir S, Baghi Yazdi M, Navabazam A . Aggressive Adenoid Cystic Carcinoma of Maxillary Sinus in a 43-Year-Old Male: Rare Case Report and Review of Literature. Case Reports in Medicine . 2017;2017:2324717. doi:10.1155/2017/2324717.

3. Mathai M, Sherubin JE, Agnihotri P, Sangeetha G. Adenoid Cystic Carcinoma of Child: A Rare Case. International Journal of Clinical Pediatric Dentistry. 2014;7(3):206-208. doi:10.5005/jp-journals-10005-1266

4. Deepak C, Subramanian J, Ravindran C (2012) . Adenoid Cystic Carcinoma of the Maxilla-A Case Report and 5 Year Follow-up. J Clin Case Rep .2:221. doi:10.4172/21657920.1000221.

5. Zhao C, Liu J-Z, Wang S-B, Wang S-C . Adenoid cystic carcinoma in the maxillary gingiva: a case report and immunohistochemical study. Cancer Biology \& Medicine 2013;10(1):52-54. doi:10.7497/j.issn.2095-3941.2013.01.009.

\section{Learning Points:}

- Adenoid cystic carcinoma (ACC) is a rare malignant tumour mainly affecting major and minor salivary glands .

- Long term survival can be achieved by surgery and radiotherapy.

- Perineural invasion is responsible for high local recurrence.

- Prognosis is dependant on solid histological type, perineural spread, distant metastasis and locoregional recurrences . 